\title{
LAS BANDAS DE CAZADORES-RECOLECTORES PORTADORAS DEL TECNOCOMPLEJO SOLUTRENSE EN EL SUROESTE DE LA PENÍNSULA IBÉRICA. LA ARTICULACIÓN SOCIAL DEL TERRITORIO
}

\section{BANDS OF HUNTERS AND GATHERERS WITH THE SOLUTREAN TECHNO-COMPLEX IN THE IBERIAN SOUTH-WEST. THE SOCIAL ARTICULATION OF THE TERRITORY}

por

\author{
VICENTE CASTAÑEDA FERNÁNDEZ
}

Este trabajo está dedicado al Prof. Enrique Vallespí, por su labor docente e investigadora y por su calidad humana.

RESUMEN

ABSTRACT

Palabras claves

Key words
Este trabajo aborda la ocupación del territorio por las sociedades de cazadores-recolectores portadoras del tecnocomplejo Solutrense en el Suroeste de la Península Ibérica.

This article undertakes the occupation of the territory for the societies of hunters-gatherers with technology Solutrean in the Southwest of the Iberian Peninsula.

Territorio, Cazadores-Recolectores, Solutrense, Suroeste de la Península Ibérica.

Territory, Hunters-Gatherers, Solutrean, Southwest of the Iberian Peninsula.

\section{LA ARTICULACIÓN SOCIAL DEL TERRITORIO EN EL SUROESTE DE LA PENÍNSULA IBÉRICA A FINALES DEL PLEISTOCENO. UNA PROPUESTA DE ANÁLISIS}

La articulación del territorio ${ }^{1}$ por parte de una sociedad es el reflejo de la formación social a la que nos estamos refiriendo. Así, no presentan los mismos intereses sobre un determinado territorio una formación social cazadora-recolectora que una clasista inicial, por poner un ejemplo totalmente contrapuesto.

Antes de comenzar a analizar la ocupación social por parte de las bandas de cazadores-recolectores en el Suroeste de la Península Ibérica, nos parece interesante aproximarnos a la biocenosis de finales del

1. Quiero agradecer los comentarios y la ayuda prestada en la elaboración de este artículo por Nuria Herrero y José Ramos. 
Pleistoceno. Este hecho nos permitirá conocer, sobre la base del registro arqueológico, los alimentos y las materias primas que la naturaleza produce, las cuales, por medio de los distintos modos de trabajo son apropiados por los hombres y las mujeres agregados en bandas con el objetivo de llevar a cabo sus tareas de producción y reproducción.

La falta de investigación sobre la reconstrucción de la biocenosis del Sur de la Península Ibérica durante el Pleistoceno, ha favorecido el traslado mimético de la secuencia glaciar alpina, sin atender a sus peculiaridades regionales. Frente a este modelo, nos parece interesante la propuesta desarrollada por Ruiz Bustos, quién para las Cordilleras Béticas plantea el dominio de unas condiciones climáticas templadas (interglaciares) durante el Pleistoceno frente a los fríos, que apenas se dejan sentir en la zona (Ruiz Bustos 1991 y 1997). Este hecho podemos constatarlo en Andalucía a finales del Pleistoceno sobre la base de la distribución de las manifestaciones simbólicas (pictóricas y grabadas) y la fauna fósil asociada a los tecnocomplejos Solutrense y Magdaleniense, en las que no se ha constatado ningún tipo de "fauna fría", sino al contrario, una fauna dominada por los cérvidos, cápridos y équidos, entre otros. Circunstancia ésta que se repite a lo largo de todo el Pleistoceno.

A pesar del dominio de unas condiciones climáticas templadas, no queremos rechazar la influencia que ejerció en estos momentos la última glaciación, que posiblemente alcanzó las condiciones climáticas mas rigurosas durante sus pulsaciones mas frías (en torno al 21.000 B.P.) en el Sur de la Península Ibérica. Así, no es descartable, que a pesar de la latitud que presenta la región, en el Sur de la Península Ibérica se produjeran los fenómenos glaciares más meridionales de toda Europa, en zonas concretas de Sierra Nevada o de la Serranía de Ronda, que todavía conserva el topónimo de Sierra de las Nieves.

Estas sociedades, para alcanzar un máximo de optimación en la apropiación de los recursos subsistenciales y con ello el desarrollo de las fuerzas productivas, recurren al nomadismo, y en definitiva a la ocupación estacional del territorio, como tendremos ocasión de comprobar más adelante. Con esta propuesta, se pretende romper con el carácter estático planteado para las sociedades del Paleolítico por la Arqueología Tradicional.

Estos territorios, frecuentados estacionalmente por medio del nomadismo, permiten plantear sobre los mismos una verdadera territorialidad. El concepto de territorio debe ser entendido como la articulación del espacio por una estructura social (Arteaga, Ramos y Roos 1998).

Las sociedades de bandas, al ser depredadoras, se apropian, mediante distintos modos de trabajo, de lo que la naturaleza produce (alimentos, materias primas,...), no llevando a cabo de esta forma una propiedad sobre los objetos de trabajo. A pesar de que no exista una propiedad real sobre los medios naturales de producción, ello no implica, como ya hemos comentado, la existencia en las sociedades de bandas de verdaderos territorios, controlados por posesión consensuada o apropiación estacional (Arteaga, Ramos y Roos 1998; Ramos et al. 1998).

Esta territorialidad puede constatarla en el ámbito arqueológico sobre la base de la distribución no solamente de los productos relacionados con la vida material, sino también con las manifestaciones simbólicas.

Este comportamiento moderno, y en definitiva esta ocupación estacional del territorio con el objetivo de apropiarse de los recursos subsistenciales producidos por los diferentes medios naturales, podemos rastrearlo en homínidos anteriores a la llegada del Homo sapiens sapiens al Sur de la Península Ibérica ${ }^{2}$ (Herrero y Castañeda, 1998). Así, si hoy día la mayoría de los investigadores rechazan la relación mimética entre registro antropológico y sistema técnico, este hecho también se debería hacer extensible al comportamiento social.

2. De este modo, en momentos avanzados del tecnocomplejo Achelense, durante lo que Vallespí denominó como Achelense Pleno para el Sur de la Península Ibérica, se empieza a constatar una serie de cambios en el registro arqueológico. Así, estos homínidos asociados antropológicamente a los últimos Homo erectus u Homo heidelbergensis, según las distintas propuestas, empiezan a tener una movilidad más marcada que en momentos anteriores, que permite, según Vallespí, alcanzar las zonas del Subbético andaluz, en sus latitudes bajas y medias, por parte de los grupos occidentales en busca de nuevas materias primas silíceas para la realización de útiles sobre lasca (Vallespí 1992, 1994). 


\section{LAS BANDAS DE CAZADORES-RECOLECTORES CON TECNOCOMPLEJOSOLUTRENSE EN EL SUROESTE DE LA PENÍNSULA IBÉRICA}

Quizá, uno de los grandes aciertos del Prof. Vallespí, que siempre guió sus investigaciones, fue proponer una secuencia paleolítica para el Sur de la Península Ibérica atendiendo al registro arqueológico y a sus peculiaridades regionales (Vallespí 1992 y 1994). Nosotros, desde la posición teórica de la Arqueología Social incidiremos en los conceptos analíticos de modo de producción, modo de vida y modo de trabajo para profundizar en el estudio de la formación social de cazadores recolectores, planteándonos como objetivo estudiar el registro arqueológico, al igual que ha puesto de manifiesto el Prof. Vallespí a lo largo de sus investigaciones, contextualizado en el Sur de la Península Ibérica, con la intención de plantear un modelo de contrastación alternativo al proporcionado para otras regiones.

A pesar de que en los últimos años la investigación ha deparado nuevos hallazgos, las bases arqueológicas de las que partimos para desarrollar este trabajo son aún muy limitadas. Sin embargo, aunque es verdad que el número de sitios ha aumentado, estos han sido tratados en su mayoría desde los parámetros del Historicismo Cultural, limitando, por su carácter, las inferencias históricas que se pueden obtener del registro arqueológico.

De esta forma, el registro arqueológico estudiado por la Arqueología Tradicional relacionado con el tecnocomplejo Solutrense del Sur de la Península Ibérica tan sólo se ha preocupado por la construcción cronocultural, donde un sitio arqueológico se relaciona con este tecnocomplejo tan sólo por la presencia/ausencia de un "fósil guía" como es un determinado foliáceo. Por el contrario, desde nuestra posición teórica, la presencia de distintos tipos de foliáceos lo relacionamos con la invención del arco y la flecha, es decir, con una herramienta de trabajo que revolucionó las técnicas de caza, ya que por primera vez el hombre no tenía que acercarse a los animales para abatirlos, sino que desde una distancia mas o menos cómoda podía realizar sus actividades cinegéticas. Junto a ello, la invención, por ejemplo, del pedúnculo y las aletas le permitiría alcanzar una gran precisión a la hora de llevar a cabo el trabajo de caza, favoreciendo de este modo un aumento de la productividad.

De este modo, pretendemos darle a la "industria lítica" un contenido distinto al que tradicionalmente se le ha supuesto, tan sólo como indicador cronocultural. Así, debemos relacionarlas con herramientas de trabajo que nos acercan a la base económica de las sociedades, a todo lo referente con el trabajo y al desarrollo de las fuerzas productivas.

El hecho de que el tecnocomplejo Solutrense haya sido identificado fundamentalmente sobre la base de unas herramientas de trabajo como son las puntas de flecha, nos ha llevado a un problema añadido. Así, la mayoría de los sitios arqueológicos localizados están en relación con los trabajos de apropiación de los recursos (altos de caza y cazaderos), dejando en un segundo plano los vinculados con la vida cotidiana.

Como es lógico, estos altos de caza van a estar relacionados con pasos naturales, tales como, los que posiblemente se localicen en la cueva de los Ojos (Cozvijar, Granada)(Toro y Almohalla 1985) y la cueva del Boquete de Zafarraya (Alcaucín, Málaga)(Barroso, Hublin y Medina 1992), o con farallones rocosos situados frente a zonas lagunares, como por ejemplo Pantano de Cubillas (Albolote, Granada)(Toro y Ramos 1985) o Peña de la Grieta (Porcuna, Jaén)(Arteaga, Ramos y Roos 1998).

Siguiendo con la línea argumental expresada con anterioridad, partimos de la premisa según la cual las bandas de cazadores-recolectores relacionadas con el final del tecnocomplejo Solutrense ocuparon el territorio por medio de frecuentaciones cíclicas y controlados por posesión consensuada o apropiación estacional sobre la base del nomadismo. Así, las zonas de montaña se ocupan durante las estaciones templadas (primavera y verano), mientras que las de valle se habitarían durante las más rigurosas (otoño e invierno), con el objetivo de evitar las rigurosidades climáticas.

Para estos momentos, esta ocupación estacional del territorio podemos explicarla desde los sitios de agregación, definidos por Conkey (1980 y 1984) y Utrilla (1994) para la Cornisa Cantábrica. Consideramos 
que ésta es una herramienta útil para explicar no solamente la conciencia social, el sistema de valores, sino también la articulación del territorio de estas bandas de cazadores-recolectores, ya que en ellos aparecen una serie de manifestaciones simbólicas característicos de la identificación social de las mismas con unos territorios determinados.

¿Qué son los sitios de agregación? Los sitios de agregación son lugares, localizados en cuevas o al aire libre, frecuentados estacionalmente por bandas de cazadores-recolectores procedentes de distintos territorios y medios naturales.

Para Cantalejo, los sitios de agregación deben relacionarse con intensas actividades sociales, posibilitando la exogamia para la regulación biológica del grupo y la ampliación de la configuración de la banda (Bate 1986), transmisión de conocimiento con relación a la tecnología y a los modos de trabajo, práctica de distintos ritos de iniciación,... (Cantalejo 1995; Cantalejo y Espejo 1998; Ramos, Cantalejo y Espejo 1999). En la misma línea, tendríamos que mencionar las apreciaciones planteadas por Arteaga, Ramos y Roos, según los cuales estas cavidades se deberían relacionar con lugares idóneos para las frecuentaciones colectivas de estas sociedades de bandas, identificándose, entre otras cosas, con relaciones parentales, para la celebración de rituales y ceremonias de "alianzas", actividades de reciprocidad cooperativa, e incluso como lugares de convergencia para la concreción de ententes territoriales, los cuales no solamente se realizan a niveles parentales sino también al superestructural con el objetivo de una conciencia social (Arteaga, Ramos y Roos 1998: 95).

Estos ententes territoriales podemos constatarlos en el Sur de la Península Ibérica sobre la base de los restos arqueológicos relacionados, no solamente con la vida material, sino también con las manifestaciones simbólicas, las cuales en el estado actual de la investigación nos ayudan en mayor medida a la definición territorial.

Estos sitios de agregación han sido identificados por Cantalejo (1995) en el Sur de la Península Ibérica a partir de cavidades tales como la de Pileta (Benaoján, Málaga), Ardales (Ardales, Málaga), e incluso Nerja (Nerja, Málaga), todas ellas vinculadas a los territorios de Andalucía Occidental y Central, para los momentos relacionados con los tecnocomplejos Solutrense y Magdaleniense (Ramos, Espejo y Cantalejo 1997; Ramos, et al. 1998).

¿Qué tienen en común estos sitios para ser identificados como lugares de agregación? Aquí, tendríamos que mencionar una serie de características tales como (Cantalejo 1995):

a) Si en estos sitios de agregación se reunían bandas procedentes de diferentes territorios, estos deben encontrarse bien comunicados con diversos medios naturales a través de distintas vías de comunicación. Así, la cueva de la Pileta, localizada en la serranía de Ronda, se relaciona con los valles y la costa atlánticamediterránea a través de los ríos Guadiaro y Guadalete, éste último a través de un largo paso estrecho que comunica la vertiente atlántica y mediterránea por medio de la Manga de Villaluenga, mientras que con el bajo valle del Guadalquivir por medio del río Corbones. Una situación muy parecida le ocurre a la cueva de Ardales, relacionada con la costa malagueña a través del río Guadalhorce.

b) Estos sitios presentan una altitud media relativamente baja, con el objetivo de evitar las rigurosidades climáticas producidas durante las pulsaciones frías acaecidas durante la última glaciación, como la ocurrida en torno al 18.000 B.P. Así, aunque como ya hemos comentado, el Sur de la Península Ibérica gozó del dominio de unas condiciones climáticas templadas (interglaciares) durante el Pleistoceno, las zonas de alta montaña debieron sufrir un clima bastante extremo.

Este hecho nos permite situar los sitios de agregación localizados en el Sur de la Península Ibérica en latitudes medias/bajas, tales como la cueva de Ardales, que se encuentra aproximadamente a unos $400 \mathrm{~m}$, o la cueva de Pileta, situada a unos $800 \mathrm{~m}$.

c) Son cavidades con grandes dimensiones, vertebradas por distintas salas y de fácil acceso por medio de grandes salones. 
d) Estos sitios presentan una amplia cronología, contrastada en los numerosos paneles bien estructurados, documentándose aportes iconográficos no simultáneos a lo largo de un período considerable. Este hecho debemos relacionarlo con una frecuentación estacional que favorecería el ir añadiendo paulatinamente distintos paneles hasta alcanzar su configuración final como lugar de agregación o como santuario colectivo, tal como lo identifica Cantalejo (1995), que al fin y al cabo nos está hablando del nomadismo, y en definitiva de las distintas frecuentaciones practicadas por estas sociedades.

e) Éstas, presentan un importante repertorio tanto material como simbólico, con diversos paneles donde se representan animales de distintos biotopos, fruto de una ocupación estacional de bandas procedentes de distintos medios naturales.

f) Los paneles se encuentran perfectamente estructurados a lo largo de las diversas salas, no documentándose de una forma aleatoria, tal como se ha puesto de manifiesto en las cuevas de Ardales y Pileta. En esta última, tendríamos que destacar la magnitud de su recorrido por las galerías principales, donde las representaciones y los lugares elegidos para los mismos van aumentando en espectacularidad, hasta llegar al escenario final situado en el Salón del Pez (Cantalejo 1995: 218).

Con estas apreciaciones, tan sólo queremos poner de manifiesto el carácter ordenado de las manifestaciones simbólicas en las distintas cavidades, las cuales no presentan en ningún caso un aspecto aleatorio. De este modo, para Ramos, Cantalejo y Espejo "las cuevas con arte tienen una organización impresionante en la distribución de los temas y signos. Ello sólo puede ser producto de una adecuación y planificación muy cuidada, que deben ser abordados en perspectiva histórica, según modelos de formas y motivos" (Ramos, Cantalejo y Espejo 1999: 169).

La figura de un gran pez como tema central se repite tanto en la cueva de Ardales (Ardales, Málaga) como en la de Pileta (Benaoján, Málaga), documentándose también en las cuevas de las Motillas (Jerez de la Frontera, Cádiz)(Giles et al. 1997 y 1998) y en Nerja (Nerja, Málaga), aunque en esta última recientes investigaciones han relacionado las manifestaciones simbólicas identificadas tradicionalmente como peces con focas (Pérez Ripoll y Raga, 1998). La figura de un pez, que no es un tema muy frecuente en las manifestaciones simbólicas de las sociedades de bandas, aparece en el Sur de la Península Ibérica como un elemento característico en diferentes cavidades, curiosamente durante el tecnocomplejo Solutrense, en el que la pesca todavía no había adquirido las proporciones de épocas posteriores como un recurso de complementación económica de la caza y la recolección.

La presencia de unos temas y unas técnicas que se repiten a lo largo de las distintas cavidades no deben ser interpretadas desde los parámetros difusionistas, sino que éstos eran conectados entre sí por medio de las frecuentaciones estacionales llevadas a cabo por las bandas de cazadores-recolectores, con el objetivo, en última instancia, de fomentar ideológicamente una conciencia social en un territorio. Así, "las pinturas rupestres plasman unas 'formas' que podrían darse en distintos nichos ecológicos, que eran conectados, sin embargo, por las frecuentaciones estacionales que tales bandas llevan a cabo"(Arteaga, Ramos y Roos 1998: 95).

De esta forma, podemos comprobar como en el Sur peninsular existía a finales del Pleistoceno Superior una homogeneidad técnica y temática en las manifestaciones simbólicas documentadas en las distintas cavidades, que se traduce en la conformación de verdaderos territorios por parte de las sociedades de bandas sobre la base de su modo de producción cazador-recolector.

Posteriormente, planteamos como hipótesis de trabajo como los grupos agregados o bandas ampliadas reunidos en estos sitios de agregación, se dividen y se dispersan por las latitudes bajas para llevar a cabo trabajos de apropiación, generalmente durante las estaciones con un clima mas riguroso.

Como hemos comentado, los sitios de agregación son una herramienta muy útil no sólo para explicar la conciencia social, el sistema de valores de estas sociedades, sino también la articulación del territorio por parte de las bandas de cazadores-recolectores. Este último apartado está escasamente desarrollado aún para estos momentos en el Suroeste de la Península Ibérica, aunque podemos esbozar una serie de líneas generales. 
Centrándonos en el territorio articulado desde el sitio de agregación de la cueva de la Pileta (Benaoján, Málaga) (Cantalejo 1995; Sanchidrián $1997^{3}$; Cantalejo y Espejo 1998) (fig. 1) que, como ya hemos comentado, se relacionaría a grandes rasgos con las Subbéticas occidentales y los valles de la actual provincial de Cádiz, nos encontramos con lugares con una clara vinculación simbólica (pequeñas cavidades o abrigos con escasas manifestaciones simbólicas, ya sean pictóricas o grabadas, y con un importante carácter monotemático), junto a otros que nos informan de los trabajos de apropiación de los recursos (altos de caza, cazaderos,...) y los de la vida cotidiana (campamentos), como expresión empírica de los modos de trabajo desarrollados por las bandas de cazadores-recolectores.

Como ya hemos tenido ocasión de comentar, la cueva de la Pileta se encuentra perfectamente comunicada con los valles y la costa atlántica-mediterránea por medio de unos pasos naturales. Sin duda alguna, éstos serían utilizados como verdaderos pasos naturales que permitirían vincular los valles y la costa con las zonas de montaña, y en definitiva con los distintos biotopos que recorren sus respectivas cuencas.

La cueva de la Pileta ha proporcionado la presencia de tres ciclos con relación a las manifestaciones simbólicas documentadas, de los cuales los dos primeros se corresponden con el denominado tecnocomplejo Solutrense, mientras que el tercero con el Magdaleniense (Cantalejo 1995; Cantalejo y Espejo 1998; Sanchidrián 1997). Este hecho nos está hablando del importante período histórico durante la cual fue frecuentada la misma.

Siguiendo el curso del río Guadalete, como vía de comunicación natural entre el valle y la montaña, nos encontramos con las cavidades del Complejo Motillas-Ramblazo (Jerez de la Frontera, Cádiz), localizado en uno de los relieves del Subbético que se encuentran aisladas de los demás, y donde se han documentado algunas cavidades asociadas al tecnocomplejo Solutrense en sus momentos finales. Así, en esta zona de montaña tendríamos que destacar la cueva del Higueral (Jerez de la Frontera, Cádiz)(Giles et al. 1997 y 1998), próxima al sitio de agregación de la cueva de Pileta y posiblemente vinculada con un alto de caza, de la cual sabemos, sobre la base de los estudios faunísticos, que fue ocupada mediante frecuentaciones cíclicas durante las estaciones de primavera a otoño (Cáceres y Anconetani 1998) por sociedades portadoras de los tecnocomplejos Solutrense Superior y Solutreogravetiense (Giles et al. 1998), circunstancia esta última que nos informa de distintos momentos de ocupación. Como podemos comprobar, el esquema se repite, las zonas de montaña de habitan durante las estaciones mas templadas.

Dentro del Complejo Motillas-Ramblazo, también tendríamos que destacar el abrigo del Bombín (Jerez de la Frontera, Cádiz), donde la presencia de algunos restos humanos, una punta de escotadura, una lasca con retoque simple y una concha de cardium edule con el interior impregnado de ocre ha llevado a sus autores a considerarlo como un lugar de enterramiento. A pesar de que este reducido número de hallazgos han sido recogidos en una prospección superficial, sus autores lo relacionan con el tecnocomplejo Solutreogravetiense (Giles et al. 1997 y 1998).

La presencia de conchas de moluscos marinos en zonas del interior no nos debe extrañar, ya que también se ha documentado en otros sitios, tales como la cueva de los Ojos (Cozvijar, Granada) (Toro y Almohalla 1985). Este hecho nos hace reflexionar sobre la importancia del nomadismo como un mecanismo efectivo a la hora de aprovechar los recursos producidos por los diferentes biotopos. A pesar de ello, estos moluscos marinos y otros productos arqueológicos pudieron llegar a esta zona como consecuencia de los complejos sistemas de complementación económica encaminados a paliar la precariedad económica estructural de la formación social de cazadores-recolectores. De esta forma, por medio de este mecanismo se podría establecer un proceso de distribución entre las diferentes bandas o unidades sociales, facilitando así la apropiación de objetos de trabajo en espacios geográficos más amplios.

3. J.L. Sanchidrián, desde un planteamiento estructuralista, realiza una propuesta de la secuencia figurativa de la cueva de la Pileta en base a un estudio estilístico. Ello, le lleva a diferenciar tres etapas (Solutrense antiguo, Solutrense reciente y Magdaleniense reciente), que al fin y al cabo nos informa de la frecuentación de la cavidad durante un período de tiempo amplio. 
La circulación de productos arqueológicos, tanto alimenticios como manufacturados, no son debidos única y exclusivamente a la movilidad de las bandas de cazadores-recolectores en su ocupación de los diferentes nichos ecológicos, sino que también pueden ser los resultados de las relaciones sociales de unos grupos con otros, donde se llevan a cabo actividades de distribución y cambio de productos. Como es lógico, el hecho de encontrarnos ante sociedades igualitarias, al menos en teoría, de bandas de cazadores-recolectores determina que la distribución, y en definitiva el consumo de los productos, también se haga de una forma igualitaria.

Pero esta circulación no solamente hace referencia a los productos, sino también a las ideas, favoreciendo que en un mismo territorio se constaten pinturas y grabados rupestres con una temática y unos estilos muy homogéneos como expresión de sus modos de vida (Arteaga, Ramos y Roos 1998; Ramos et al. 1998).

En el tramo medio del río Guadalete se han podido constatar la presencia de algunos sitios al aire libre tales como La Escalera 3 (Arcos de la Frontera, Cádiz) (Giles et al. 1997 y 1998) o Llanos de Don Pedro (Arcos de la Frontera, Cádiz) (Giles et al. 1997 y 1998) y en pequeñas cavidades como la cueva del Higueral de Sierra Valleja (Arcos de la Frontera, Cádiz), situándose esta última a unos 170 m s.n.m. en la margen Sur de la sierra Valleja y con orientación al río Majaceite, desde donde se domina un amplio territorio hacia el interior de la depresión del río Guadalete caracterizado por la gran biodiversidad existente y varias ocupaciones adscritas a los tecnocomplejos Solutrense Superior y Solutrense Superior Evolucionado o Solutreogravetiense (Giles et al. 1997 y 1998), interpretándose la misma como un alto de caza relacionado con la caza y el procesado de cérvidos (Cáceres 1998 y 1999).

Pero junto al modo de trabajo de la caza, debemos recordar el importante papel que debió jugar la apropiación de los recursos vegetales por medio de la recolección. A pesar de ello, en Andalucía se desconoce aún la importancia que pudo tener la recolección en las sociedades de bandas, dado que los escasos sitios excavados pertenecientes a estas fechas no cuentan aún con estudios arqueobotánicos. Así, si estamos proponiendo unas condiciones templadas para el Sur de la Península Ibérica, por lo menos no tan frías como las que presentarían latitudes mas elevadas, estaremos igualmente de acuerdo con la importancia, al igual que ocurre con la fauna, de unos recursos vegetales estacionales, que adquirirían su máxima expresión a finales de las estaciones de verano y principios del otoño.

Para Giles y su equipo (Giles et al. 1997 y 1998), estos sitios arqueológicos localizados en la cuenca media del río Guadalete no solamente estarían en relación con la apropiación de recursos alimenticios, sino también silíceos para la fabricación de las herramientas de trabajo.

Los trabajos de investigación desarrollados en la actual costa y valles del centro de la banda atlántica de Cádiz, que también estaban bien comunicada con las áreas de montaña por medio de los ríos GuadaleteMajaceite y Barbate, han permitido documentar algunos sitios al aire libre, tales como La Fontanilla (Conil de la Frontera, Cádiz)(Ramos, Castañeda y Gracia 1995) o Casa de Postas (Conil de la Frontera, Cádiz)(Ramos et al. 1994), y algunos pequeños abrigos situados en determinadas crestas rocosas de Sierra Momia, tales como Cuevas de Levante (Benalup, Cádiz)(Ripoll López, Mas y Perdigones 1993). Todos ellos localizados en sitios idóneos para llevar a cabo procesos de trabajo depredadores, pudiéndose relacionar La Fontanilla con trabajos domésticos, y en definitiva vinculados con la vida cotidiana (Ramos, Castañeda y Gracia 1995).

Quizá, el gran handicap que presentan estos sitios al aire libre o en pequeños abrigos localizados en la banda atlántica de Cádiz sea la ausencia de restos orgánicos debido al tipo de depósito en el que se documentan los productos arqueológicos. Este hecho dificulta las inferencias históricas obtenidas a partir del registro arqueobotánico y arqueofaunístico, sesgando la información.

Por otra parte, como ya hemos tenido ocasión de comprobar, el Campo de Gibraltar también estaría relacionado con el sitio de agregación de la cueva de Pileta (Benaoján, Málaga) por medio de una vía de comunicación como fue la cuenca del río Guadiaro. A pesar de que las investigaciones en esta comarca son aún muy incipientes con relación a estos momentos históricos (Castañeda 2001), en los últimos años se han documentado en la bahía de Algeciras algunos sitios al aire libre tales como Torre Almirante (Algeciras, 
Cádiz)(Castañeda y Herrero 1998) o en pequeñas cavidades, tales como cueva "S" (también denominada cueva Sewell)(Gibraltar)(Giles et al. 1996: 14).

Estos sitios arqueológicos vinculados a la vida cotidiana y a la apropiación documentados en el Suroeste de la Península Ibérica, presentan diferentes herramientas de trabajo atendiendo a las actividades productivas desarrolladas en cada una de ellas. Así, como es lógico, no se documentan los mismos útiles en un sitio relacionado con un alto de caza del interior, un campamento o un sitio costero, ya que en ellos para momentos sincrónicos se han desarrollado actividades productivas subsistenciales, en algunos aspectos, distintas.

Esta circunstancia ha llevado a graves errores a la Arqueología Tradicional, centrada en un simple cómputo normativo, al identificar como tecnocomplejos diferentes a las evidencias materiales relacionables con distintos modos de trabajo y dejadas por las mismas bandas de cazadores-recolectores en su nomadismo cíclico de costa-valle-montaña. Así, la propuesta planteada por el normativismo, según la cual los momentos avanzados del tecnocomplejo Solutrense puede ser sincrónicos a las primeras etapas del Magdaleniense, ha sido recientemente explicado por Arteaga, Ramos y Roos (1998: 93), desde la posición teórica de la Arqueología Social, como pertenecientes a distintos modos de trabajo propios de contemporáneos modos de vida pertenecientes al modo de producción caracterizado en los distintos medios naturales del Sur de la Península Ibérica.

Pero en estos territorios de las actuales campiñas de la banda atlántica de Cádiz y del Campo de Gibraltar, no solamente nos encontramos con sitios relacionados con la apropiación o con la vida cotidiana, sino también con una clara vinculación simbólica, relacionados con los denominados "santuarios locales" (Cantalejo 1995; Cantalejo y Espejo 1998) tales como la cueva de las Motillas (Jerez de la Frontera, Cádiz)(Santiago 1989; Giles et al. 1997 y 1998), cuevas de la Manga de Villaluenga (Villaluenga del Rosario, Cádiz) (Giles et al. 1995-1996, 1997 y 1998), el Tajo de las Figuras (Benalup, Cádiz) (Ripoll López, Mas y Torra 1991), la cueva del Moro (Tarifa, Cádiz) (Bergmann 1996; Mas et al. 1997) o la cueva de las Palomas (Facinas, Cádiz)(Breuil y Burkitt 1929; Santiago 1979-1980), cuyas manifestaciones simbólicas han sido puestas en duda recientemente (Sanchidrián 1994b; Cortés et al. 1996).

La frecuentación cíclica que hemos ido analizando a lo largo de nuestro trabajo, también podemos inferirla a partir de manifestaciones simbólicas documentadas en algunos "santuarios locales" en el territorio vertebrado por la cueva de la Pileta. Aquí, hemos hallado la presencia de varios estilos en una misma cavidad, tal como ocurre en las cavidades de las Motillas (Jerez de la Frontera, Cádiz)(Cantalejo y Espejo 1998). Este hecho unido a las evidencias arqueológicas documentadas en la misma ha permitido a Giles y a su equipo relacionarlo con un lugar de agregación (Giles et al. 1997: 401), aunque mas bien la presencia de distintas figuras y estilos se deba más a la dilatada ocupación, como lugar estratégico para llevar a cabo las tareas depredadoras, que como un lugar de agregación de diferentes bandas.

Todos estos sitios, relacionados con una clara vinculación simbólica, se corresponden con cuevas o abrigos de reducidas dimensiones, donde se documenta un reducido número de manifestaciones simbólicas, tanto pictóricas como grabadas, y una temática muy monótona (Cantalejo 1995). De esta forma, se aleja de los grandes paneles y de la gran representación de temas localizados en los sitios de agregación.

Dentro de los dos estilos artísticos atribuidos al tecnocomplejo Solutrense, es sin duda alguna el estilo II, que presenta un mayor desarrollo en cuanto a su variedad, forma, temática, el que está mejor documentado en los territorios vertebrados por la cueva de la Pileta, tal como se constata en las cavidades de las Motillas (Jerez de la Frontera, Cádiz)(Santiago 1989), las cavidades de la Manga de Villaluenga (Villaluenga del Rosario, Cádiz)(Giles et al. 1997 y 1998) o la cueva del Moro (Tarifa, Cádiz)(Bergmann 1996; Mas et al. 1995 y 1997).

Desde una propuesta materialista, las manifestaciones simbólicas documentadas en las cavidades del Sur de la Península Ibérica son explicadas como expresión ideológica de los modos de vida de las sociedades de bandas que ocuparon estos territorios, a partir de los cuales se pueden obtener inferencias tanto del modo 
de producción como del modo de reproducción de las mismas (Ramos Muñoz et al. 1998; Ramos, Cantalejo y Espejo 1999). Con ello se han planteado nuevas vías de explicación al margen de lo puramente estilístico.

Junto a estos hechos, los estudios sobre las manifestaciones simbólicas representadas, las técnicas empleadas en su ejecución, el canon de las figuras y sus planteamientos orgánicos también nos deben ayudar, como ya hemos tenido ocasión de comentar, a definir territorialidades. Así, estamos de acuerdo con Arteaga, Ramos y Roos cuando afirman "que las bandas cazadoras-recolectoras dejan en los territorios frecuentados no solamente lugares significativos de la apropiación de sus recursos, sino también símbolos expresos de su identificación social con tales territorios" (Arteaga, Ramos y Roos 1998: 95), infiriéndose a partir de ellos una conciencia colectiva producto de una ideología común.

A diferencia de los planteamientos realizados desde propuestas tales como el Historicismo Cultural, consideramos que las pinturas rupestres no se encuentran desconectadas de los territorios de caza y recolección, y en definitiva del modo de producción cazador-recolector, debiéndose estudiar por lo tanto en relación a éstos y en definitiva incluyéndose dentro de los territorios productivos de las bandas de cazadores-recolectores.

Estas evidencias, tanto materiales como simbólicas, localizadas en el Suroeste de la Península Ibérica estarían encaminados, como ya hemos comentado con anterioridad, con la identificación social de tales territorios con determinadas bandas. A pesar de ello, este modelo no podemos entenderlo como un sistema cerrado, ya que una banda de cazadores-recolectores no tiene porqué frecuentar el mismo sitio de agregación todos los años, pudiendo de esta forma variar.

En conclusión, pensamos que la Arqueología Social plantea una perspectiva válida para el estudio de la formación social de cazadores-recolectores, donde ésta es mas que una simple cultura o adaptación al medio (Ramos 1999).

\section{BIBLIOGRAFÍA}

ARTEAGA, O., RAMOS, J., y ROOS, A.M. (1998): “La Peña de la Grieta (Porcuna, Jaén). Una nueva visión de los cazadores-recolectores del mediodía atlántico-mediterráneo desde la perspectiva de sus modos de vida y de trabajo en la cuenca del Guadalquivir", en J.L. SANCHIDRIÁN y M.D. SIMÓN., eds.: Las culturas del Pleistoceno Superior en Andalucía: 75-109. Patronato de la Cueva de Nerja. Málaga.

BARROSO, C.; HUBLIN, J.J. y MEDINA, F. (1992): "Zafarraya y el remplazamiento de los neandertales por el hombre moderno anatómicamente en Europa Occidental (objetivos del proyecto general de investigación y resumen de los resultados obtenidos en las campañas de 1990 a 1993", Investigaciones Arqueológicas de Andalucía: 1985-1992. Proyectos: 229-238. Huelva.

BATE, L.F. (1986): "El modo de producción cazador-recolector, o la economía del salvajismo", Boletín de Antropología Americana 13. México.

BERGMANN, L. (1996): "Los grabados paleolíticos de la cueva del Moro (Tarifa, Cádiz). El arte rupestre del Paleolítico más meridional de Europa”, Almoraima 16: 9-26. Mancomunidad de Municipios del Campo de Gibraltar.

BREUIL, H. y BURKITT, M. (1929): Rock Paintings of southern Andalusia. A description of a Neolithic and Cooper Age art group. Clarendon Press. Oxford.

CÁCERES, I. (1998): “Agentes tafonómicos y económicos de los grupos de cazadores-recolectores de la Cueva de Higueral de Sierra Valleja (Cádiz)", Revista Atlántica-Mediterránea de Prehistoria y Arqueología Social. Vol. I: 57-76. Servicio de Publicaciones de la Universidad de Cádiz. Cádiz.

- (1999): Estudio tafonómico y paleoeconómico de la cueva de Higueral de sierra Valleja. Servicio de Publicaciones de la Universidad de Cádiz. Cádiz.

CÁCERES, I. y ANCONETANI, P. (1998): "Procesos tafonómicos del nivel Solutrense de la cueva de Higueral de Motillas (Cádiz)”, Zephyrus, 50: 37-52. 1997 Universidad de Salamanca. Salamanca. 
VICENTE CASTAÑEDA FERNÁNDEZ

CANTALEJO, P. (1995): “Arte paleolítico del sur peninsular. Las manifestaciones costeras y los santuarios de interior”, en RAMOS, J. et al., El Paleolítico Superior Final del río Palmones (Algeciras, Cádiz). Un ejemplo de la tecnología de las comunidades especializadas de cazadores-recolectores. Instituto de Estudios Campogibraltareños. Algeciras.

CANTALEJO, P. y ESPEJO, M.M. (1998): “Arte rupestre paleolítico del sur peninsular. Consideraciones sobre los ciclos artísticos de los grandes santuarios y sus territorios de influencia”, Revista AtlánticaMediterránea de Prehistoria y Arqueología Social I: 77-96. Servicio de Publicaciones de la Universidad de Cádiz.

CASTAÑEDA, V. (2000): Las sociedades de bandas de cazadores-recolectores en Andalucía. Tesis Doctoral Inédita. Universidad de Cádiz. Cádiz.

- (2001): "El estudio de las sociedades del Paleolítico en el Campo de Gibraltar. Una historia olvidada", Jornadas de Historia del Campo de Gibraltar. Instituto de Estudios Campogibraltareños. Octubre de 2000. Gibraltar. ALMORAIMA 25: 37-47. Algeciras.

CASTAÑEDA, V. y HERRERO, N. (1998): "Torre Almirante (Algeciras, Cádiz). Un nuevo asentamiento al aire libre de cazadores-recolectores especializados en el Sur de la Península Ibérica”, Caetaria 2: 11-24. Museo Municipal. Ayuntamiento de Algeciras. Algeciras.

CONKEY, M. (1980): "The identification of prehistoric hunter-gatherer aggregation sites: the case of Altamira", Current Anthropology 21, 5: 609-630.

- (1984): "To find ourselves: Art and Social Geography of Prehistoric Hunter Gatherers", en SCHRIRE, C. ed.: Past and Present in Hunter-Gatherer Studies. Academic Press. Nueva York.

CORTÉS, M.; MUÑOZ, V.E.; SANCHIDRIÁN, J.L. y SIMÓN, M.D. (1996): El Paleolítico en Andalucía. La dinámica de los grupos predadores en la Prehistoria andaluza. Ensayo de síntesis. Córdoba.

GILES, F.; SANTIAGO, A.; GUTIÉRREZ, J.M.; MATA, E. y AGUILERA, L. (1996): "Nuevas evidencias de Paleolítico Superior en el extremo sur europeo. Estudio de materiales depositados en el Gibraltar Museum", Caetaria 1: 11-18. Museo Municipal. Ayuntamiento de Algeciras. Algeciras.

GILES, F.; SANTIAGO, A.; GUTIÉRREZ, J.M. y MATA, E. (1997): "Las comunidades del Paleolítico Superior en el extremo sur de Andalucía Occidental. Estado de la cuestión", en BALBÍN, R. y BUENO, P. eds.: II Congreso de Arqueología Peninsular. Paleolítico y Epipaleolítico. Tomo I: 383-403. Fundación Rei Afonso Henriques. Zamora.

GILES, F.; GUTIÉRREZ, J.M.; SANTIAGO, A. y MATA, E. (1998): “Avance al estudio sobre poblamiento del Paleolítico Superior en la cuenca media y alta del río Guadalete (Cádiz)”, en SANCHIDRIÁN, J.L. y SIMÓN, M.D. eds.: Las culturas del Pleistoceno Superior en Andalucía: 111-140. Patronato de la Cueva de Nerja. Málaga.

HERRERO, N. y CASTAÑEDA, V. (1998): "Los Neandertales : los grandes marginados de la evolución humana", Revista Atlántica Mediterránea de Prehistoria y Arqueología Social, I: 33-56. Servicio de Publicaciones de la Universidad de Cádiz. Cádiz.

MAS, M.; RIPOLL, S.; MARTOS, J.A.; PANIAGUA, J.P.; RAMÓN, J. y BERGMANN, L. (1995): "Estudio preliminar de los grabados rupestres de la Cueva del Moro (Tarifa, Cádiz) y el arte paleolítico del Campo de Gibraltar", Trabajos de Prehistoria 52, 2: 61-81. Madrid.

MAS, M.; RIPOLL, S.; CACHO, C.; PANIAGUA, J.P.; LÓPEZ, J.R.; MARTOS, J.A. y BERGMANN, L. (1997): "Los grabados paleolíticos de la Cueva del Moro (Tarifa, Cádiz)", en FULLOLA, J.M. y SOLER, N. eds.: El món mediterrani després del Pleniglacial (18.000-12.000 B.P): 355-363. Serie Monográfica, 17. Museo de Arqueología de Catalunya. Gerona.

PÉREZRIPOLL, M. y RAGA, J.A. (1998): "Los mamíferos marinos en la vida y en el arte de la prehistoria de la Cueva de Nerja", en SANCHIDRIÁN, J.L. y SIMÓN, M.D. eds.: Las culturas del Pleistoceno Superior en Andalucía: 251-275. Patronato de la Cueva de Nerja. Málaga.

RAMOS MUÑOZ, J. (1999): Europa Prehistórica. Cazadores y Recolectores. Ed. Sílex. 
RAMOS MUÑOZ, J.; CASTAÑEDA, V. y GRACIA, F.J. (1997): "El asentamiento al aire libre de La Fontanilla (Conil de la Frontera, Cádiz). Nuevas aportaciones para el estudio de las comunidades de cazadoresrecolectores especializados en la Banda Atlántica de Cádiz”, Zephyrus XLVIII: 269-288. Universidad de Salamanca. Salamanca.

RAMOS MUÑOZ, J.; ESPEJO, M. y CANTALEJO, P. (1998): “La Cueva de Ardales (Málaga). Enmarque histórico regional y aportaciones a la movilidad organizada de las comunidades de cazadores-recolectores especializados", en SANCHIDRIÁN, J.L. y SIMÓN, M.D. eds.: Las culturas del Pleistoceno Superior en Andalucía. Patronato de la Cueva de Nerja: 197-216. Nerja. Málaga.

RAMOS MUÑOZ, J.; CANTALEJO, P. y ESPEJO, M. (1999): "El arte de los cazadores-recolectores como forma de expresión de los modos de vida. Historiografía reciente y crítica a las posiciones eclécticas de la posmodernidad", Revista Atlántica Mediterránea de Prehistoria y Arqueología Social II: 151-177. Servicio de Publicaciones de la Universidad de Cádiz. Cádiz.

RAMOS MUÑOZ, J.; CASTAÑEDA, V.; PÉREZ, M. y LAZARICH, M. (1994): "Las ocupaciones humanas de la Prehistoria Reciente de la campiña litoral y banda atlántica de Cádiz", Gibraltar during the Quaternary. AEQUA Monografías 2: 71-90. Gibraltar.

RAMOS MUÑOZ, J.; CANTALEJO, P.; ESPEJO, M. y CASTAÑEDA, V. (1998): “El arte de los cazadoresrecolectores como expresión de sus modos vida", Revista de Arqueología 206: 10-19. Madrid.

RIPOLL LÓPEZ, S.; MAS, M. y TORRA, G. (1991): "Grabados paleolíticos en la Cueva del Tajo de las Figuras (Benalup, Cádiz)”, Espacio, Tiempo y Forma. Serie I. Prehistoria y Arqueología, IV: 111-126. Madrid.

RIPOLL LÓPEZ, S.; MAS, M. y PERDIGONES, L. (1993): “Actuaciones de urgencias en las cuevas de Levante y Cubeta de La Paja (Sierra Morena, Benalup, Cádiz)”, Anuario Arqueológico de Andalucía. 1991. II Actividades Sistemáticas: 105-110. Sevilla.

RUIZ BUSTOS, A. (1991): "Hacia una cronología del Cuaternario continental ibérico. Cuestiones básicas a considerar", Raña 10, I-VIII. Sevilla.

_- (1997): "Características bioestratigráficas y paleoecológicas que implican los mamíferos en las cuencas de las Cordilleras Béticas", Cuaternario Ibérico: 283-296. Madrid.

SANCHIDRIÁN, J.L. (1994): “Arte Paleolítico de la zona meridional de la Península Ibérica”, Complutum 5: 163-195. Madrid.

(1997): "Propuesta de la secuencia figurativa en la Cueva de la Pileta", en FULLOLA, J.M. y SOLER, N. eds.: El món mediterrani després del Pleniglacial (18.000-12.000 BP). Serie Monográfica 17: 411-430. Museo de Arqueología de Catalunya. Gerona.

SANTIAGO, J.M. (1979-1980): "La cueva de las Palomas en el arte paleolítico del Sur de España", Boletín del Museo de Cádiz II: 5-11. Cádiz.

_ (1989): “Avance al estudio del arte parietal paleolítico de la Cueva de Las Motillas (Cádiz)”, Páginas Revista de Humanidades 1. Jerez de la Frontera.

TORO, I. y ALMOHALLA, M. (1985): "Descubrimiento de industria del Paleolítico Superior en la Provincia de Granada. El Yacimiento Solutrense de la cueva de los Ojos (Cozvíjar, Granada)", XVII Congreso Nacional de Arqueología: 97-104. Madrid.

UTRILLA, P. (1994): "Campamentos-base, cazaderos y santuarios. Algunos ejemplos del paleolítico peninsular", Monografías del Museo y Centro de Investigaciones de Altamira. Homenaje al Dr. González Echegaray n ${ }^{\circ}$ 17: 97-113. Madrid.

VALLESPÍ, E. (1992): "Las industrias Achelenses en Andalucía: Ordenación y Comentarios”, Spal 1: 61-78. Sevilla.

_ (1994): "El Bajo Guadalquivir en el Paleolítico Inferior y Medio peninsular", Homenaje al Dr. Joaquín González Echegaray. Museo y Centro de Investigación de Altamira. Monografía n 17: 13-16. Ministerio de Cultura. Santander. 


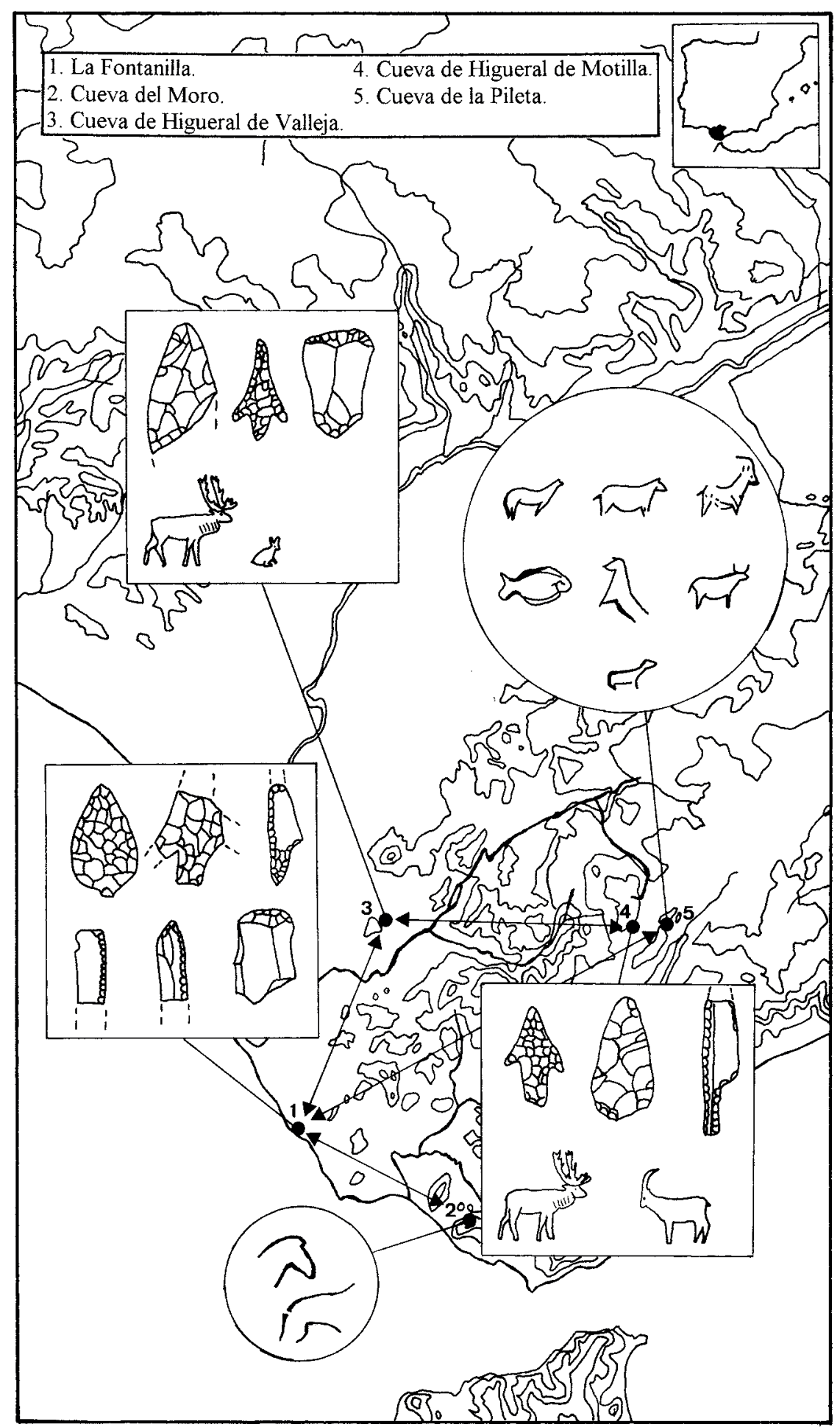

Figura 1: Modelo ideal sobre la frecuentación del territorio por bandas de cazadores-recolectores. 